\title{
Avaliação de Ferramentas de Autoria Gratuitas para produção de Objetos de Aprendizagem no padrão SCORM
}

\author{
Title: Evaluation of Free Authoring Tools for SCORM Learning Objects Production \\ Paulo Eduardo Battistella \\ UnA-SUS - Universidade Aberta do SUS - Núcleo SC \\ Hospital Universitário - HU \\ Universidade Federal de Santa Catarina - UFSC \\ paulo@telemedicina.ufsc.br \\ Aldo von Wangenheim \\ INCoD - Instituto Nacional para Convergência Digital \\ Centro Tecnológico - CTC \\ Universidade Federal de Santa Catarina - UFSC \\ awangenh@inf.ufsc.br
}

\begin{abstract}
Resumo Este artigo apresenta a avaliação de um conjunto de seis ferramentas de autoria para produção de objetos de aprendizagem no padrão SCORM. As ferramentas são avaliadas de acordo com dois critérios: usabilidade e utilidade. A usabilidade é medida através da aplicação de dois métodos de avaliação heurística a cada uma das ferramentas após a produção de um objeto de aprendizagem com conteúdo pré-definido. A utilidade é medida através da avaliação da utilidade dos objetos de aprendizagem produzidos com cada ferramenta através da aplicação de uma terceira avaliação heurística específica. Para possibilitar uma avaliação comparativa tanto das ferramentas como dos objetos de aprendizagem produzidos, foi tomado um objeto de aprendizagem de referência, produzido profissionalmente no contexto de uma grande iniciativa de ensino à distância, que foi reproduzido o mais fielmente possível com cada uma das ferramentas. As ferramentas de autoria foram previamente sistematicamente selecionadas a partir de um universo de 14 ferramentas gratuitas.
\end{abstract}

Palavras-Chave: Ferramentas de Autoria, Objetos de Aprendizagem, Usabilidade, Utilidade.

\begin{abstract}
This paper presents the evaluation of a set of six authoring tools for SCORM-conform learning objects. The tools are validated according to two criteria: usability and effectiveness. Usability is measured through the application of two distinct sets of usability heuristics after the production of a learning object with a predefined content. Utility is measured through the evaluation of the quality of the learning objects produced with each tool through the application of a third, specific, set of heuristics. In order to allow a comparative evaluation of both, the tools and the produced learning objects, a reference learning object was taken, which was previously professionally produced in the context of a large distance learning initiative, and which was then reproduced as faithfully as possible with each of the tools. The authoring tools were previously systematically selected from an universe of 14 free tools.
\end{abstract}

Keywords: Authoring tools, Learning objects, Usability, Effectiveness 


\section{Introdução}

Iniciativas no setor público de Educação como UAB [1], a UnA-SUS [2] e as instalações de Ambientes Virtuais de Ensino Aprendizado (AVEA) para apoio ao ensino presencial praticadas em várias Instituições de Ensino Superior (IES) Federais, para sua sustentabilidade a médio e longo prazo, dependem de muitos fatores associados ao material didático, como por exemplo Repositórios de Objetos de Aprendizagem (OAs), AVEAs de boa qualidade, amplo acesso a ambientes de aprendizado por parte dos alunos e, principalmente, conteúdo com qualidade, atratividade e usabilidade. Um desses fatores é o custo associado à produção de conteúdo de qualidade. Hoje, principalmente em iniciativas como UAB e UnASUS, este material é produzido por equipes multidisciplinares compostas por, de um lado Especialistas do Domínio e, de outro lado, Designers Instrucionais e Designers Gráficos (DI\&DG). Essas equipes de DI\&DG são hoje equipes altamente treinadas e que utilizam ferramentas de autoria de conteúdo comerciais sofisticadas e de alto custo e com uma longa curva de aprendizagem. Isto significa que o DI\&DG não só representa um Recurso Humano (RH) dispendioso, mas também representa custos na forma de licenças de software.

Atualmente (ano base: 2011) muitos professores universitários interessados em desenvolver material de ensino de qualidade e destinado aos AVEAs dependem de equipes de DI\&DG, o que é um considerável fator de custo e cria gargalos no processo de produção. Isto, por um lado, dificulta a maior disseminação do uso de AVEAs e, por outro lado, exige um investimento contínuo em atividades de editoração de conteúdo para iniciativas públicas de Ensino a Distância (EaD) como UAB e UnASUS.

Para que a produção contínua de conteúdo tanto em AVEAs das IESs, bem como iniciativas públicas de EaD se tornem um processo sustentável, de baixo custo e uma parte natural do processo de preparo de aulas em universidades, é necessário que se ofereça aos Especialistas de Domínio uma paleta de Ferramentas de Autoria (FAs) para suportar esse processo, além da necessidade de uma possível integração destas ferramentas aos AVEAs.

Entendemos que essas FAs devam atender os seguintes requisitos:

1. Oferecer níveis de usabilidade para permitir que professores de áreas não tecnológicas e com pouca ou nenhuma familiaridade com informática e editoração de conteúdo se encontrem em condições de produzir conteúdo após um treinamento curto ou mesmo de forma autodidata [3].
2. Ser gratuitas e preferencialmente de software livre para permitir que cada IES possa customizá-las se necessário.

3. Produzir OAs com níveis de qualidade de apresentação e usabilidade comparáveis aos de conteúdo produzido por ferramentas comerciais profissionais.

4. Oferecer um nível de integração adequado com AVEAs e Repositórios de OAs de uso comum, de forma que o processo de produção, oferta, reuso e extensão de OAs seja facilitado e adequado ao uso por leigos em administração de AVEAs e OAs.

Nesse sentido, existe a necessidade de se realizar uma revisão sistemática das FAs para $\mathrm{EaD}$ sendo oferecidas, tanto do ponto de vista de sua usabilidade, como do ponto de vista da utilidade dos OAs produzidos.

O presente artigo está estruturado da seguinte forma: Objetivos, Métodos de Avaliação, Trabalhos Relacionados, Ferramentas Avaliadas, Avaliação e Conclusão.

\section{Objetivos}

O objetivo deste trabalho é avaliar seis FAs sistematicamente selecionadas em [4], tanto do ponto de vista de sua usabilidade quanto do ponto de vista da sua utilidade. Para tanto empregamos duas metodologias de análise heurística para avaliação de usabilidade das FAs. E uma metodologia heurística para análise da utilidade de seus OAs. As FAs analisadas são: CourseLab, eXeLearning, HotPotatoes, Microsoft LCDS, MyUdutu e Xerte.

Este artigo dá continuidade às pesquisas iniciadas em [4], onde foram analisadas 14 FAs de acordo com os seguintes requisitos: (a) código aberto/software gratuito, (b) integração dos OAs produzidos a um AVEA no padrão SCORM e (c) integrabilidade semântica desses OAs. Em [4] foram selecionadas seis FAs satisfazendo critérios mínimos, as quais são o foco do presente artigo.

Entendemos que a produção de OAs de forma sustentável necessita de um processo transparente aos professores. Neste sentindo, a pesquisa apresentada em [4] corresponde com a seleção de FAs, no presente artigo buscamos avaliar as FAs e em futuro trabalho buscaremos a integração da FA melhor avaliada para integração ao AVEA.

\section{Métodos}

A pesquisa avalia as FAs através do desenvolvimento de OAs similares um OA produzido pela equipe de 
DI\&DG da UAB/UFSC no âmbito do Projeto UnaSUS/UFSC. Essa equipe utiliza ferramentas de edição de OAs de caráter profissional e aplicadas em larga escala na UAB. Neste contexto foi selecionado um OA o qual é considerado o padrão-ouro onde os resultados da aplicação das FAs aqui avaliadas são comparados. Sendo denominado de Objeto de Aprendizagem-Referência (OA$\mathrm{R})$.

A avaliação das Ferramentas de Autoria está dividida em duas formas: avaliação de Usabilidade (eficiência) das Ferramentas de Autoria e avaliação da Utilidade (efetividade) dos OAs produzidos.

\subsection{Metodologia de Avaliação das Ferramen- tas de Autoria}

Para avaliar as Ferramentas de Autoria quanto à usabilidade é aplicada a metodologia de avaliação heurística. $\mathrm{Na}$ avaliação heurística é utilizada as 10 Heurísticas de Nielsen[5] e os Critérios Ergonômicos de Bastien e Scapin[6].

Para ambas as heurísticas, o processo empregado é de primeiramente utilizar as FAs para o desenvolvimento de um OA o mais fiel possível ao OA-R e, a seguir, aplicar as heurísticas a cada uma das ferramentas.

\subsubsection{As 10 Heurísticas de Nielsen}

Neste método as interfaces são avaliadas através de 10 critérios, oferecendo guidelines específicas para medir a usabilidade das ferramentas propostas. Os critérios utilizadas são: (1) visibilidade do status do sistema, (2) compatibilidade entre o sistema e o mundo real, (3) controle e liberdade para o usuário, (4) consistência e padrões, (5) prevenção de erros, (6) reconhecimento no lugar da lembrança, (7) flexibilidade e eficiência no uso, (8) projeto minimalista e estético, (9) auxilia os usuários a reconhecer, diagnosticar e recuperar erros e (10) ajuda e documentação.

\subsubsection{Critérios Ergonômicos de Bastien e Scapin}

Bastien e Scapin[6] propõem critérios ergonômicos visando o desenvolvimento de métodos e ferramentas com o objetivo de atribuir fatores humanos na concepção e avaliação das Interfaces Homem-Máquina (IHM). Estes critérios são vistos como recurso para definir e operacionalizar as dimensões de usabilidade. Os critérios propostos pelos autores são: (1) condução, (2) carga de trabalho, (3) controle explícito, (4) adaptabilidade, (5) gestão de erros, (6) consistência, (7) significado de código e denominações e (9) compatibilidade.

\subsection{Metodologia de Avaliação dos Produtos Gerados (OAs)}

Para avaliação da utilidade dos OAs produzidos é necessária uma métrica objetiva e reprodutível. Para esse fim são utilizados os critérios de utilidade para OAs determinados em $[7,8,9]$.

As pesquisas citadas fazem parte de um grupo de pesquisadores de OA que em [8] apresenta os resultados de pesquisa de quatro repositórios públicos aplicando as tecnologias de clustering e algoritmos de mineração de dados para obter características de conteúdos dos OAs.

Os critérios de Utilidades propostos pelo resultado das pesquisas citadas estão divididos no seguinte aspecto: (1) Funcionalidade, (2) Usabilidade, (3) Eficiência e (4) Reutilização.

\subsubsection{Objeto de Aprendizagem de Referência}

Para utilizar um OA como padrão-ouro a pesquisa empregou conteúdo público do Curso de Especialização em Saúde da Família da UnA-SUS/UFSC produzido pela equipe de DI\&DG por meio de ferramentas comerciais, como Adobe Flash, Adobe Dreamweaver, Adobe Photoshop e Corel Draw. Veja na figura 1 o OA-referência (OA-R).

O OAR é considerado como padrão-ouro pelas seguintes características: (a) proposto por um especialista do domínio com experiência na proposição de cursos em IES, (b) projetado em formato digital pelo DI especializado, (c) implementando pelo DG, o qual apresenta formação técnica e experiência na área.

O curso de Especialização em Saúde da Família da Una-SUS no contexto da universidade possui 19 módulos, onde cada módulo possui de 5 a 15 OAs produzidos pelo DI\&DG, juntamente com os Especialistas do Domínio. Portanto, ao nomear o OA-R como referência da pesquisa, é considerado uma fração do conjunto de OAs produzidos para o curso da universidade.

No artigo, a definição de OA é fundamentada nas pesquisas de Relvão[10], onde se apresenta revisão bibliográfica do assunto. Os OAs produzidos no experimento relatado neste artigo seguem o padrão Sharable Content Object Reference Model(SCORM) e o padrão de metadados LOM - Learning Object Metadata. O OA-R empregado na pesquisa apresenta granularidade de nível 2.

No curso de especialização, dois requisitos nãofuncionais foram seguidos, o primeiro quanto ao nível da granularidade 2 e o segundo quanto a apresentação do conteúdo em única página, contendo objetivos, conteúdo da aula e uma atividade auto-avaliativa. 


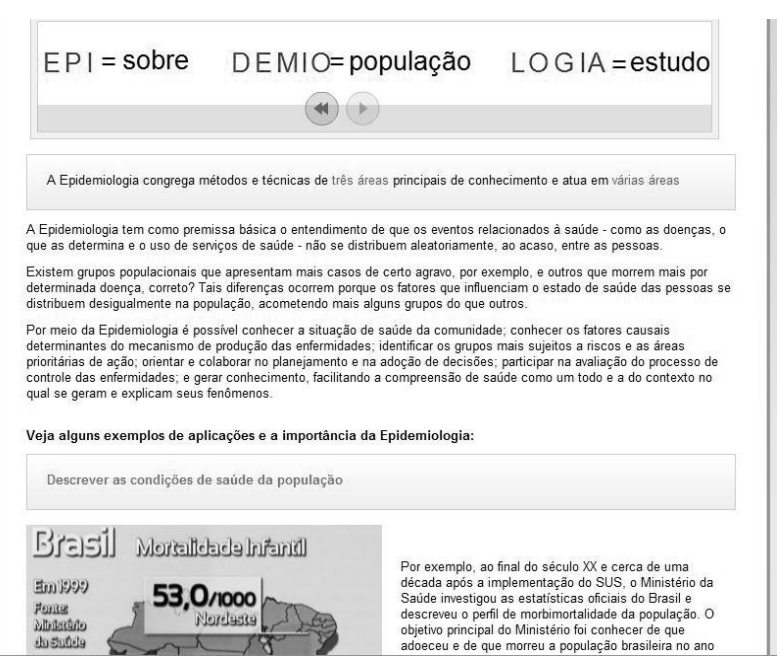

Figura 1: Objeto de Aprendizagem de Referência (OA-R)

\subsection{Quantificação da Avaliação}

Para reduzir a subjetividade da avaliação é utilizada uma escala com 5 graus para os métodos acima.

\begin{tabular}{|c|l|}
\hline Grau & \multicolumn{1}{c|}{ Descrição } \\
\hline 0 & Não aplicável \\
\hline 1 & Não atende os requisitos \\
\hline 2 & Atende cerca da metade dos requisitos \\
\hline 3 & Atende a maior parte dos requisitos \\
\hline 4 & Atende plenamente os requisitos \\
\hline
\end{tabular}

Tabela 1: Escala de Avaliação

\section{Trabalhos Relacionados}

A pesquisa de Downes[11] levanta questões relacionadas à dificuldade de desenvolvimento de materiais digitais por professores e o alto custo associado a profissionais. Contudo, é enfatizada a importância dos OAs para o ensino à distância. As Ferramentas de Autoria permitem a produção de OAs pelos próprios professores, sem exigir conhecimento profissional em DI\&DG.

A pesquisa de Boyle[12] enfatiza a importância da reutilização dos OAs, contudo ressalta a necessidade dos OAs serem independentes entre si, ou seja, devem respeitar a granularidade. O autor também propõe um framework para autoria de OAs na linguagem de programação Java. No caso das Ferramentas de Autoria os professores são conduzidos a produzirem suas aulas de forma independente, onde cada conteúdo gerado corresponde a um OA.

Em [21] apresenta um método de avaliação de ferramentas de aprendizagem para definir a compatibilidade das ferramentas ao padrão SCORM. A pesquisa avaliou
10 ferramentas, dividindo-as em três grupos principais: (a) ferramentas de empacotamento de conteúdo de aprendizagem, (b) ferramentas de autoria e (c) ferramentas de gestão de conteúdo de aprendizagem. $\mathrm{O}$ método possui duas categorias, formando ao total 11 subcategorias, onde cada item possui uma nota, sendo multiplicada pelo peso, que varia entre 1 a 5 . Ao final cada ferramenta possui um índice de classificação.

Assim como os autores a cima classificam os critérios de avaliação no contexto somente tecnológico, na pesquisa de [22] apresenta em área correlacionada 34 critérios para avaliação da qualidade de Repositórios de Objetos de Aprendizagem (ROA). Nestas pesquisas são enfatizados os requisitos tecnológicos, mas não apresentam critérios quanto a usabilidade, reusabilidade e Interfaces Homem-Máquina (IHM).

Em [13], propõem uma FA no padrão SCORM, seguindo em duas linhas de pesquisa. A primeira é o desenvolvimento da FA com formato amigável e a segunda busca aprimorar a reutilização dos OAs com Repositórios Digitais.

A pesquisa apresentada em [3] avalia uma FA considerando dois aspectos, o primeiro relacionado os cinco anos de utilização da FA e o segundo leva em consideração a eficiência da FA para produção do OA. A ferramenta apresentada não necessita de desenvolvedores para produção dos objetos de aprendizagem, sendo considerado ponto positivo da pesquisa. Os autores justificam esta vantagem relacionando as pesquisas de Murray, Ainsworth, e Blessing [20], onde as FA normalmente exigem o conhecimento e acompanhamento de desenvolvedores para produção dos OAs.

O presente artigo, aborda a criação de OAs de modo diferente da pesquisa apresentada em [3,13], as quais são utilizadas tecnologias existentes evitando implementações de novos softwares e oferece a possibilidade de seleção de ferramenta com código aberto para futura integração em AVEAs.

Em [24] é proposto um método para avaliação de usabilidade de software de automação industrial. Na pesquisa os autores utilizam as 10 Heurísticas de Nielsen e também as Normas ISO/IEC 9126 e ISO 9241-11. Estas Normas buscam avaliar a usabilidade do software por meio da eficiência, eficácia e satisfação dos usuários.

Em [25] é apresentado um método para avaliação de ferramentas de apoio a aprendizagem na disciplina de matemática e jogos pedagógicos, baseados em aspectos de usabilidade da Norma ISO 9126 e em aspectos pedagógicos.

Nas duas pesquisas citadas a cima as normas ISO são utilizadas para avaliação das ferramentas, porém está implícito que será formado um grupo de usuários para 
avaliar as ferramentas, fator que não ocorre necessariamente com a avaliação heurística. Portanto, ao propor um método para avaliação das ferramentas para apoiar a aprendizagem levamos em consideração que nem sempre professores terão a sua disposição um grupo de professores para testar e avaliar as ferramentas.

Em [26] é proposto um método para avaliação de OAs formado por três critérios, com variação da pontuação entre 0 a 10. Esta avaliação possui critérios genéricos permitindo classificar os OAs quanto ao uso: Individual ou Social, quanto à: Reflexão ou Não-Reflexão, e quanto à: Experiência ou Informação.

Contudo, neste método é possível verificar a seguinte limitação, permitir uma avaliação em apenas três critérios, desconsiderando aspectos como os pedagógicos, os tecnológicos e os de reusabilidade.

\section{Ferramentas Avaliadas}

A seguir são apresentadas brevemente as Ferramentas de Autoria. Na figura 2 é possível visualizar o melhor caso (a) e o pior caso (b) alcançados utilizando as FAs. Para observar as seis FAs acesse o link http://www.lapix.ufsc.br/index.php/fat-evaluation-offree-authoring-tools.

\subsection{CourseLab}

A CourseLab[14] é uma Ferramenta de Autoria de software livre desenvolvida para produção de Objetos de Aprendizagem, tendo semelhança com ferramentas de Apresentação como Microsoft PowerPoint. A ferramenta está disponível para download na versão 2.4 , ano base 2011, e para instalação do executável no formato Desktop.

Os desenvolvedores da FA disponibilizam manual integrado e instalador no formato wizard. Também existem recursos como animações, vídeos, diferentes formatos de textos, botões, questionários, entre outros. Permite a programação de ações entre objetos, por exemplo, ao clicar em determinada animação, a ferramenta gera ações como informar um texto na tela, executar um som ou vídeo, mas não permite a edição do HTML dos OAs.

\section{2 eXe Learnig}

A eXe Learning[15] é uma Ferramenta de Autoria de código aberto para criação de Objetos de Aprendizagem em HTML que roda localmente em navegador Web e disponibiliza aos usuários dispositivos do tipo texto de leitura, questões de múltipla escolha, questões de verdadeiro ou falso, applet Java e artigos WikiBooks.

A FA disponibiliza sete templates editáveis, um OA de exemplo descrevendo como utilizar a ferramenta, criação de iDevices e edição do HTML dos OAs. A ferramenta apresenta layout semelhante ao Moodle.

A ferramenta não existem conhecimento em programação, permitindo gerar sites estáticos a partir dos OAs. Contudo, a funcionalidade principal é a produção de OAs para utilização em LMS.

\subsection{HotPotatoes}

A HotPotatoes[16] é a Ferramenta de Autoria de software livre no formato desktop, sendo especializada em atividades. As atividades estão divididas em Atividades Interativas de Múltiplas Escolhas, Resposta Curta, Palavras Cruzadas, Caça Palavras, Correspondência e Preenchimento de Lacunas.

Ao criar uma atividade não é possível inserir páginas com textos, imagens e animações, porém estes recursos são disponíveis para uma única página. Contudo, a FA permite agrupar em um único pacote diversas atividades.

O formato da FA é reconhecido automaticamente pelo Moodle, sendo uma opção para usuários sem interesse no padrão SCORM.

\subsection{Microsoft LCDS}

LCDS[17] é a Ferramenta de Autoria gratuita da $\mathrm{Mi}$ crosoft para criação de OAs. Assim como a CourseLab, é instalada no formato Desktop e oferece aos usuários um total de vinte recursos técnicos do tipo texto, imagens, animação e vídeo.

Nesta ferramenta o template para produção dos OAs é pré-definido e estático. Também permite a produção em sequência de páginas, não permitindo a alteração do tamanho das páginas.

A FA disponibiliza manual do usuário integrado ao software e na língua inglesa. A ferramenta não permite edição do HTML dos OAs e não apresenta botão para produção do OA no formato SCORM, sendo necessário a leitura do manual do usuário para obter estas informações.

\subsection{MyUdutu}

A MyUdutu[18] é uma Ferramenta de Autoria Web e utiliza o conceito de computação nas nuvens. Esta ferramenta possui características similares aos sites de redes sociais. O cadastro no site é gratuito e permite aos usuários a alteração dos templates da ferramenta.

Entre as FAs pesquisadas neste trabalho, a MyUdutu é a única ferramenta utilizada sem necessidade de instalação ou configuração. Os recursos oferecidos pela ferramenta estão visíveis aos usuários, não exigindo navegação entre páginas para localizá-los. O processo de produção dos OAs segue algumas etapas, em que cada usuário 
"monta" uma funcionalidade do objeto, e ao final das etapas o OA está concluído.

\subsection{Xerte}

A Xerte[19] é a Ferramenta de Autoria de código aberto desenvolvida em PHP contendo recursos HTML, ActionScritps, inclusão de Google Maps, Wikipédia e vídeos do YouTube.

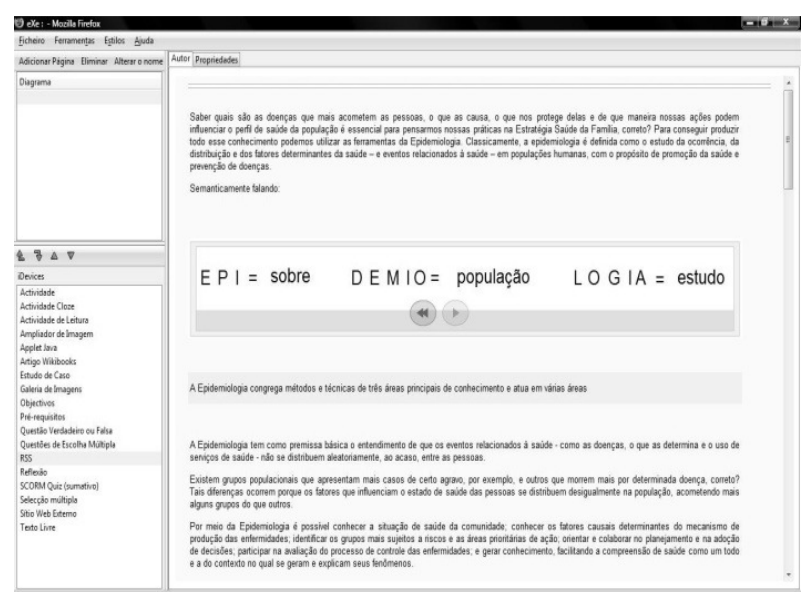

(a) eXe Learning
A FA exige conhecimento prévio em programação HTML para resultados simples, porém exige conhecimento de ActionScript para resultados mais complexos, como calcular resultados de jogos de perguntas.

Para usuários não familiarizados com programação Web existe um assistente para criação de conteúdo. Porém exige um administrador $W e b$ para instalar a FA.

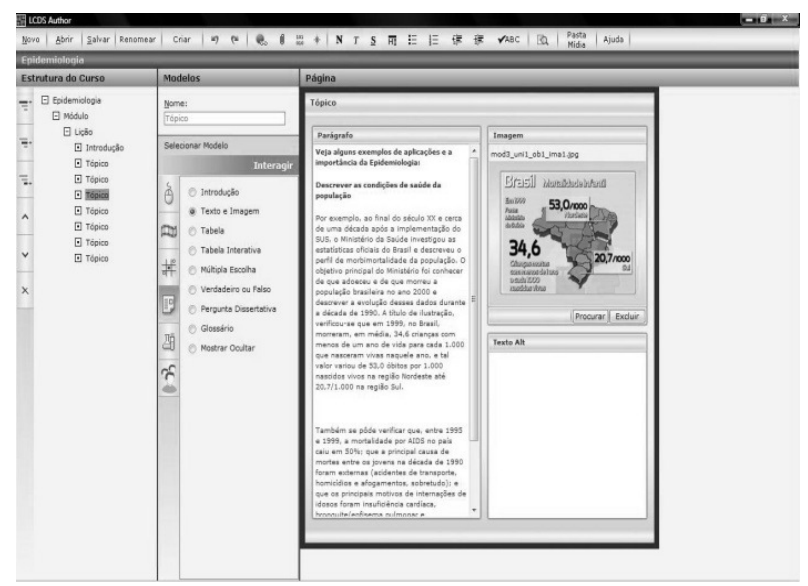

(b) Microsoft LCDS

Figura 2: Interface das Ferramentas de Autoria no melhor caso (a) e pior caso (b) apresentando conteúdo do OA-R.

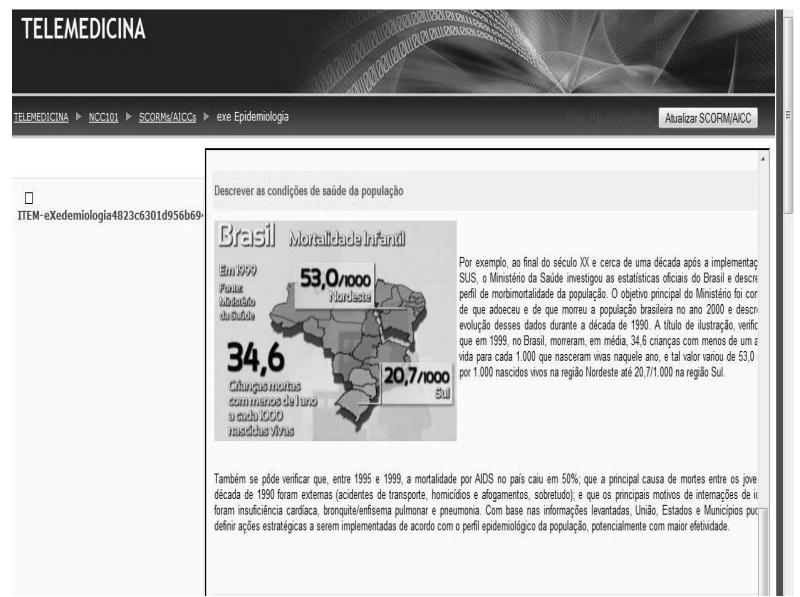

(a) eXe Learning

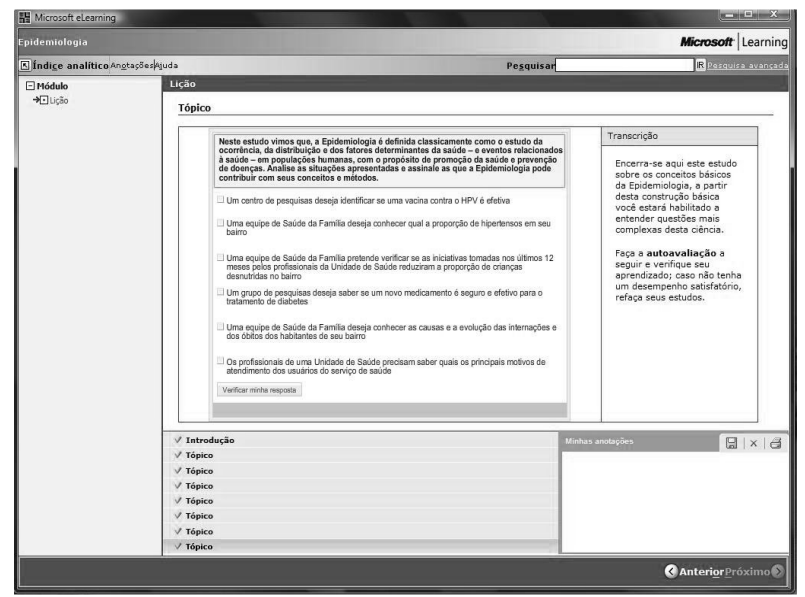

(b) Microsoft LCDS

Figura 3: Objetos de Aprendizagem no melhor caso (a) inserido no Moodle e no pior caso (b) visualizado no player da Ferramenta de Autoria. 


\section{Avaliação}

$\mathrm{Na}$ avaliação heurística cada avaliador não detecta plenamente os problemas de usabilidade existentes em um software. Em média, um avaliador detecta $35 \%$ dos problemas e cinco avaliadores detectam $75 \%$ dos possíveis problemas[23].

No presente artigo as avaliações foram realizadas por um único avaliador e desenvolvedor dos OAs produzidos pelas FAs. Neste caso, o avaliador possui conhecimento prévio em programação e experiência com as FAs apresentadas em [4]. Para redução da subjetividade são utilizados três métodos avaliativos (seção 3 ), formando ao total 58 subcategorias com notas entre 0 e 4 .

Para o contexto do artigo, a realização das avaliações por meio de um único avaliador não apresenta problemas de confiabilidade, uma vez que a presente avaliação busca selecionar uma FA para uma futura integração entre FA e AVEA.

$\mathrm{Na}$ avaliação cada FA possui uma nota conforme a avaliação heurística utilizada. Por exemplo, na avaliação de Nielsem, contendo 10 critérios, uma FA pode receber nota máxima de 40 pontos, na avaliação dos Critérios Ergonômicos, contendo 8 critérios, uma FA pode receber nota máxima de 32 pontos e na avaliação de Utilidade dos OAs, contendo 40 critérios, uma FA pode receber até 160 pontos.

A seguir são apresentados os resultados gerais da aplicação dos três métodos avaliativos e os resultados específicos obtidos individualmente pelas FAs. Visualize também na figura 3 o melhor caso (a) e o pior caso (b) alcançados com a produção dos OAs. Para observar todos os OAs produzidos acesse o link http://www.lapix.ufsc.br/index.php/fat-evaluation-offree-authoring-tools.

\subsection{Resultados gerais}

A seguir são descritos os principais pontos fortes e fracos encontrados através da avaliação de Nielsen e apresentados na tabela 2 .

Os critérios comentados a seguir são abordados dentro dos itens que alcançaram as maiores ou menores notas pelas ferramentas.

No critério visibilidade do status do sistema, onde o software busca deixar o usuário informado quanto a situação do sistema, ou seja, apresenta feedback adequando ao usuários, a $L C D S$ possui única estrutura hierárquica na barra de ferramentas, onde os botões "criar", "abrir", "salvar" e "renomear" estão dispostos ao lado de botões para formatação de textos. Eventualmente um usuário pode utilizar estas opções erroneamente, ou simplesmente clicar no botão e o sistema não informar caso a ação do botão não seja executada. No caso da Xerte, não é possível visualizar o OA sem a opção preview, pois os assest são inseridos sequencialmente em árvore de diretórios e o usuário busca informar as posições " $\mathrm{X}$ " e "Y" para cada asset da página.

No critério compatibilidade entre sistema e mundo real, onde o sistema apresenta linguagem clara e não técnica a Xerte obteve baixo índice. Por outro lado, a Course Lab apresenta recursos intuitivos, onde é possível o usuário compreender a funcionalidade antes de optar pelo recurso, por exemplo, ao selecionar a barra de ferramentas a opção Textboxes o usuário visualiza os formatos das caixas de texto disponíveis nesta opção.

No critério controle e liberdade para o usuário, onde muitas vezes o usuário escolhe uma opção errada e deseja desfazer a operação a eXe Learning é a ferramenta com maior adequação para este requisito. Por exemplo, ao inserir um recurso na página do OA, é possível visualizar um menu simplificado com botões de "confirmar", "editar" e "excluir". Além da ferramenta permitir edição no fonte da página, em HTML.

No critério de consistência e padrões, onde ações disponíveis seguem um padrão bem definido, por exemplo na MyUdutu onde o padrão de cores nas abas auxilia a delimitação das funcionalidades e disponibiliza na tela apenas os recursos referentes a aba selecionada, diferente da $L C D S$ onde a barra de ferramentas possui funcionalidades diferentes e que poderiam estar melhor organizadas em grupo e subgrupo.

No critério prevenção de erros as FAs atenderam plenamente ou a maior parte dos requisitos. Um exemplo desta fato é o freqüente questionamento do sistema ao deletarmos uma animação, imagem ou páginas vinculadas ao OA.

No critério reconhecimento no lugar de lembrança, onde as FAs são intuitivas não exigindo ao usuário treinamentos exaustivos para dominar as funcionalidades da ferramenta. No caso da Xerte este é um ponto fraco.

No critério flexibilidade e eficiência de uso as FAs são avaliadas quanto a movimentação dos assets na página do OA, apresentação do conteúdo no formato do OA$\mathrm{R}$ e se os recursos oferecidos pelas FAs não influenciam negativamente no conteúdo. Por exemplo, ao inserir uma animação a ferramenta $L C D S$ apresenta tamanho fixo limitando a visualização desta animação. 


\begin{tabular}{|c|c|c|c|c|c|c|}
\hline 10 Heurísticas & $\begin{array}{l}\text { Course } \\
\text { Lab }\end{array}$ & $\begin{array}{c}\text { exe } \\
\text { Lear- } \\
\text { ning }\end{array}$ & $\begin{array}{l}\text { Hot } \\
\text { Pota- } \\
\text { toes }\end{array}$ & LCDS & $\begin{array}{l}\text { My } \\
\text { Udutu }\end{array}$ & Xerte \\
\hline Visibilidade do status do sistema & 4 & 4 & 4 & 3 & 4 & 3 \\
\hline Compatibilidade entre sistema e mundo real & 4 & 4 & 2 & 1 & 3 & 1 \\
\hline Controle e liberdade para o usuário & 4 & 3 & 2 & 1 & 2 & 1 \\
\hline Consistência e padrões & 3 & 4 & 3 & 2 & 3 & 3 \\
\hline Prevenção de erros & 4 & 4 & 3 & 3 & 4 & 4 \\
\hline Reconhecimento no lugar da lembrança & 3 & 4 & 2 & 2 & 2 & 1 \\
\hline Flexibilidade e eficiência de uso & 3 & 4 & 2 & 1 & 3 & 1 \\
\hline Projeto minimalista e estético & 4 & 3 & 2 & 2 & 4 & 4 \\
\hline $\begin{array}{l}\text { Auxiliar os usuários a reconhecer, diagnosti- } \\
\text { car e recuperar erros }\end{array}$ & 3 & 4 & 3 & 1 & 3 & 4 \\
\hline Ajuda e documentação & 4 & 4 & 3 & 3 & 3 & 4 \\
\hline Total & 36 & 38 & 26 & 19 & 31 & 26 \\
\hline Percentual & $90,00 \%$ & $95,00 \%$ & $65,00 \%$ & $47,50 \%$ & $77,50 \%$ & $65,00 \%$ \\
\hline
\end{tabular}

Tabela 2: Resultado da aplicação das 10 Heurísticas de Nielsen [5]

\begin{tabular}{|c|c|c|c|c|c|c|}
\hline Critérios Ergonômicos & $\begin{array}{l}\text { Course } \\
\text { Lab }\end{array}$ & $\begin{array}{c}\text { exe } \\
\text { Learning }\end{array}$ & $\begin{array}{c}\text { Hot } \\
\text { Potatoes }\end{array}$ & LCDS & $\begin{array}{c}\text { My } \\
\text { Udutu }\end{array}$ & Xerte \\
\hline Condução & 3 & 4 & 3 & 1 & 3 & 2 \\
\hline Carga de trabalho & 4 & 4 & 3 & 2 & 2 & 2 \\
\hline Controle explícito & 3 & 3 & 2 & 1 & 2 & 2 \\
\hline Adaptabilidade & 3 & 3 & 1 & 1 & 2 & 3 \\
\hline Gestão de erros & 4 & 4 & 3 & 3 & 4 & 4 \\
\hline Consistência & 4 & 4 & 3 & 1 & 3 & 3 \\
\hline Significado de código e denominações & 4 & 4 & 3 & 2 & 3 & 3 \\
\hline Compatibilidade & 4 & 4 & 3 & 1 & 4 & 2 \\
\hline Total & 29 & 30 & 21 & 12 & 23 & 21 \\
\hline Percentual & $90,63 \%$ & $93,75 \%$ & $65,63 \%$ & $37,50 \%$ & $71,88 \%$ & $65,63 \%$ \\
\hline
\end{tabular}

Tabela 3: Resultados da avaliação pelos Critérios Ergonômicos segundo [6]

No critério auxiliar os usuários a reconhecer diagnosticar e recuperar erros, as FAs contemplam a maior parte ou plenamente este critério, porém a $L C D S$ obteve nota baixa por não apresentar opções para exportar os OAs produzidos pela FA para o padrão SCORM ou outros padrões onde o usuário necessite reutilizar em um ROA, LMS ou como uma simples página web.

No critério ajuda e documentação todas as FAs apresentam manual de usuário e a eXeLearning, por exemplo, disponibiliza um OA integrado a ferramenta com objetivo exemplificativo.
No critério consistência, onde o sistema mantém coerência no projeto as ferramentas CourseLab, eXeLearning, HotPotatoes, MyUdutu e Xerte atenderam a maior parte do critério, no caso da $L C D S$ a estrutura ergonômica apresentada pela FA dificultou a produção similar ao OA$\mathrm{R}$.

No critério significado de código e denominações as FAs alcançaram resultados bons, porém a $L C D S$ apresentou apresenta razoável, sendo influenciado pela falta de informação quanto a produção do $\mathrm{OA}$ no padrão SCORM. Por outro lado a CousreLab e eXe Learning 
apresentam denominações claras.

No critério compatibilidade, relativo a compatibilidade do sistema com as expectativas do usuário, a eXeLearning, CourseLab e MyUdutu apresentaram maior adequação ao critério.

Na tabela 4 é possível visualizar os resultados obtidos na avaliação dos OAs e verificar que os OAs produzidos com eXe Learning e CourseLab obtiveram melhores resultados, com $91,88 \%$ e $90,63 \%$ respectivamente.

$\mathrm{Na}$ característica Funcionalidade eXe Learning e CourseLab atenderam a maior parte dos critérios. Esta característica permitiu melhor avaliar a semelhança funcional do conteúdo com o OA-R.

$\mathrm{Na}$ característica Usabilidade as Ferramentas de Autoria HotPotatoes, Microsoft LCDS, MyUdutu e Xerte obtiveram baixo resultado por prejudicar a legibilidade do conteúdo em comparação ao OA-R.

$\mathrm{Na}$ característica de Eficiência todas as ferramentas alcançaram bons resultados. Os OAs produzidos não possuíam diferenças entre formato de arquivos, comunicação com o servidor. Porém a ferramenta $L C D S$ não executou no Moodle. Os experimentos foram feitos com os navegadores Internet Explorer 8 e Firefox 3.5.

No caso da característica de Reutilização os recursos disponíveis pelas FAs influenciaram na forma final do conteúdo. Por exemplo, a separação e apresentação do conteúdo não foram as mesmas entre as ferramentas.

\subsection{CourseLab}

A CourseLab possui flexibilidade no layout, sendo a segunda ferramenta mais próxima do OA-R. A ferramenta obteve $90 \%$ nas 10 Heurísticas de Nielsen e 90,63\% dos Critérios Ergonômicos de Bastien e Scapin. Na avaliação de Utilidade do OAs apresentadas em [9], o objeto produzido pela ferramenta obteve $90,63 \%$.

A ferramenta possui recurso que não foram utilizados na produção do OA, porque o OA-R forneceu as imagens e arquivos flash's.

\section{3 eXe Learning}

A eXe Learning obteve o melhor resultado entre as Ferramentas de Autoria com 95\% para as 10 Heurísticas de Nielsen e 93,75\% para os Critérios de Ergonomia de Bastien e Scapin. Na avaliação da Utilidade do OA produzido obteve $91,88 \%$.

A Ferramenta de Autoria possui semelhança ao Moodle. Não houve problema para desenvolver o OA, sendo necessário utilizar a opção de edição em HTML para alinhamento das imagens.

\subsection{HotPotatoes}

A HotPotatoes é uma Ferramenta de Autoria desenvolvida para elaboração de atividades. $\mathrm{Na}$ avaliação, foi desenvolvido OA com granularidade 1, sendo apenas uma atividade. Este OA é baseado na atividade do OA-R.

O OA não alcançou o objetivo de layout, semelhante ao OA-R, mas pode ser considerado uma alternativa para OA com objetivos exclusivos de atividades.

$\mathrm{Na}$ avaliação das Ferramentas de Autoria, a HotPotatoes obteve $65 \%$ para as 10 Heurísticas de Nielse e 65,63\% para os Critérios de Ergonomia de Bastien e Scapin. Para mensurar a Utildiade do OA apresentadas em [9], o OA produzido obteve $69,38 \%$.

\subsection{Microsoft LCDS}

A Microsoft $L C D S$ obteve pior resultado, por não permitir flexibilidade na disposição dos Assets. A Ferramenta de Autoria não alcançou o objetivo de criação do semelhante ao OA-R. E também o OA não rodou no Moodle com IE-Internet Explorer 8 e Firefox 3.5.

A ferramenta obteve nas avaliações Heurísticas $47,50 \%$ para Nielsen e 37,50\% para Bastien e Scapin. Na avaliação de Utilidade de OA apresentadas em [9], o OA obteve $36,25 \%$.

\subsection{MyUdutu}

A MyUdutu permite, através de site $W e b$, a criação do $\mathrm{OA}$ com edição do template padrão. O OA foi criado em vários slides, diferente do CourseLab e eXe Learning onde os OAs foram produzidos em única página.

A ferramenta obteve $77,50 \%$ e $71,88 \%$ na avaliação das 10 Heurísticas e Critérios de Ergonomia, respectivamente. Quanto a utilidade do OA produzido, a ferramenta descaracterizou a forma do OA e obteve 58,13\%. Na figura 3(e) é possível visualizar o OA produzido.

\subsection{Xerte}

A Xerte, assim como Microsoft LCDS e MyUdutu, não permite a produção do OA em única página. Descaracterizando do OA-R quanto ao layout, contudo não prejudicando o conteúdo. Porém as animações flash's não executaram de modo satisfatório.

$\mathrm{Na}$ avaliação propostas por Nielsen e por Bastien e Scapin a Ferramenta de Autoria obteve 65\% e 65,63\% respectivamente. $\mathrm{Na}$ avaliação da utilidade propostas em[9] o OA obteve $63,75 \%$. Apesar do valor adquirido, o objeto obteve baixo valor quanto a característica de usabilidade. 


\begin{tabular}{|c|c|c|c|c|c|c|c|}
\hline \multicolumn{2}{|c|}{ Utilidade dos OAs } & $\begin{array}{l}\text { Course } \\
\text { Lab }\end{array}$ & $\begin{array}{l}\text { eXe } \\
\text { Lear- } \\
\text { ning }\end{array}$ & $\begin{array}{l}\text { Hot } \\
\text { Potatoes }\end{array}$ & LCDS & $\begin{array}{l}\text { My } \\
\text { Udutu }\end{array}$ & Xerte \\
\hline \multicolumn{8}{|c|}{ Características de Funcionalidade } \\
\hline \multirow[t]{9}{*}{ Adequação } & Correção com os objetivos & 4 & 3 & 3 & 1 & 2 & 3 \\
\hline & $\begin{array}{l}\text { Consistência pedagógica dos } \\
\text { OAs com a audiência }\end{array}$ & 4 & 3 & 3 & 2 & 3 & 2 \\
\hline & $\begin{array}{l}\text { Consistência pedagógica dos } \\
\text { OAs com o estilo cognitivo }\end{array}$ & 4 & 4 & 4 & 2 & 3 & 2 \\
\hline & Conteúdo suficiente & 4 & 4 & 2 & 1 & 2 & 2 \\
\hline & Complementação do conteúdo & 3 & 3 & 2 & 1 & 2 & 2 \\
\hline & Granularidade do conteúdo & 4 & 4 & 1 & 2 & 3 & 2 \\
\hline & Vigência do conteúdo & 4 & 4 & 3 & 2 & 3 & 3 \\
\hline & Confiabilidade das fontes & 4 & 4 & 2 & 2 & 3 & 2 \\
\hline & Pertinência dos meios & 4 & 4 & 1 & 1 & 1 & 2 \\
\hline Exatidão & Precisão do conteúdo & 3 & 3 & 3 & 1 & 2 & 3 \\
\hline Interoperabilidade & Dependência do software & 4 & 4 & 3 & 1 & 2 & 3 \\
\hline \multirow[t]{5}{*}{ Conformidade } & Metadado padronizado & 3 & 4 & 4 & 1 & 3 & 3 \\
\hline & Completude do padrão & 4 & 3 & 4 & 1 & 3 & 3 \\
\hline & $\begin{array}{l}\text { Correção do conteúdo do } \\
\text { metadado }\end{array}$ & 3 & 4 & 4 & 1 & 3 & 2 \\
\hline & Clareza do conteúdo & 3 & 4 & 2 & 1 & 3 & 3 \\
\hline & $\begin{array}{l}\text { Coerência pedagógica dos } \\
\text { metadados }\end{array}$ & 3 & 3 & 3 & 0 & 2 & 3 \\
\hline Recuperabilidade & Restabelece estado & 3 & 3 & 2 & 0 & 2 & 2 \\
\hline \multicolumn{8}{|c|}{ Características de Usabilidade } \\
\hline \multirow[t]{3}{*}{ Compreensibilidade } & Clareza do conteúdo & 4 & 4 & 2 & 1 & 2 & 1 \\
\hline & $\begin{array}{l}\text { Correção quanto ao conteúdo } \\
\text { e gramática }\end{array}$ & 4 & 4 & 4 & 3 & 2 & 3 \\
\hline & Aportes de meios & 4 & 4 & 2 & 1 & 2 & 2 \\
\hline \multirow{2}{*}{$\begin{array}{l}\text { Facilidade de A- } \\
\text { prendizagem }\end{array}$} & Organização do Conteúdo & 3 & 3 & 3 & 2 & 1 & 1 \\
\hline & Desenho padronizado & 4 & 4 & 4 & 2 & 2 & 1 \\
\hline \multirow[t]{5}{*}{ Atração } & Fomenta a colaboração & 3 & 3 & 3 & 1 & 1 & 2 \\
\hline & Fomenta a interação & 4 & 4 & 4 & 2 & 1 & 2 \\
\hline & Fomenta a criatividade & 4 & 3 & 1 & 1 & 1 & 2 \\
\hline & Fomenta a motivação & 4 & 4 & 2 & 1 & 1 & 1 \\
\hline & Uniformidade & 3 & 4 & 3 & 3 & 3 & 2 \\
\hline Operabilidade & $\begin{array}{l}\text { Adaptabilidade ao estilo de } \\
\text { aprendizagem }\end{array}$ & 3 & 3 & 2 & 0 & 1 & 1 \\
\hline Conformidade & $\begin{array}{l}\text { Conformidade com o padrão } \\
\text { de usabilidade }\end{array}$ & 4 & 4 & 2 & 1 & 2 & 2 \\
\hline
\end{tabular}




\begin{tabular}{|c|c|c|c|c|c|c|c|}
\hline \multicolumn{2}{|c|}{ Utilidade dos OAs } & $\begin{array}{l}\text { Course } \\
\text { Lab }\end{array}$ & $\mathbf{e X e}$ & $\begin{array}{l}\text { Hot Pota- } \\
\text { toes }\end{array}$ & LCDS & $\begin{array}{c}\text { My } \\
\text { Udutu }\end{array}$ & Xerte \\
\hline \multicolumn{8}{|c|}{ Características de Eficiência } \\
\hline Tempo de resposta & $\begin{array}{l}\text { Troca de dados entre servidor e } \\
\text { cliente }\end{array}$ & 4 & 4 & 3 & 3 & 3 & 4 \\
\hline $\begin{array}{l}\text { Utilização de recur- } \\
\text { sos }\end{array}$ & Eficiência no armazenamento & 4 & 4 & 3 & 3 & 3 & 4 \\
\hline $\begin{array}{l}\text { Facilidade de } \\
\text { Instalação }\end{array}$ & Suporte para instalação & 4 & 4 & 4 & 3 & 4 & 4 \\
\hline Coexistência & Dependência do hardware & 4 & 4 & 3 & 1 & 4 & 4 \\
\hline \multicolumn{8}{|c|}{ Características de Reutilização } \\
\hline \multirow[t]{4}{*}{$\begin{array}{l}\text { Reutilização de con- } \\
\text { teúdo }\end{array}$} & $\begin{array}{l}\text { Independência de aspectos } \\
\text { religiosos }\end{array}$ & 4 & 4 & 4 & 2 & 3 & 3 \\
\hline & $\begin{array}{l}\text { Independência de aspectos } \\
\text { gráficos }\end{array}$ & 3 & 3 & 2 & 1 & 2 & 3 \\
\hline & Independência de aspectos éticos & 3 & 3 & 3 & 2 & 3 & 4 \\
\hline & $\begin{array}{l}\text { Independência de aspectos } \\
\text { políticos }\end{array}$ & 4 & 4 & 4 & 2 & 3 & 4 \\
\hline \multirow{3}{*}{$\begin{array}{l}\text { Desenho Reutilizá- } \\
\text { veis }\end{array}$} & Autonomia & 4 & 4 & 3 & 1 & 2 & 3 \\
\hline & Dispersão de objetivos & 3 & 4 & 3 & 1 & 3 & 3 \\
\hline & $\begin{array}{l}\text { Separação do conteúdo e da } \\
\text { apresentação }\end{array}$ & 3 & 4 & 1 & 1 & 2 & 4 \\
\hline \multicolumn{2}{|r|}{ Total } & 145 & 147 & 111 & 58 & 93 & 102 \\
\hline \multicolumn{2}{|r|}{ Percentual } & $90,63 \%$ & $91,88 \%$ & $69,38 \%$ & $36,25 \%$ & $58,13 \%$ & $63,75 \%$ \\
\hline
\end{tabular}

Tabela 4: Avaliação da Utilidade dos OAs

\section{Conclusões}

No artigo, foram avaliadas as Ferramentas de Autoria CourseLab, eXeLearning, HotPotatoes, Microsoft LCDS, MyUdutu e Xerte do ponto de vista de sua usabilidade e a utilidade dos Objetos de Aprendizagem produzidos. Na pesquisa foram produzidos os OAs similares a um OA-R desenvolvido pela equipe de DI\&DG da IES.

O OA-R faz parte do conteúdo público do curso de Especialização em Saúde Pública da Una-SUS contendo ao total 19 módulos com cerca de 5 a 15 OAs por módulo.

Levando em consideração os métodos avaliativos de Nielsen, Critérios Ergonômicos e Utilidade do OA, são apresentadas as seguintes características das FAs:

1. CourseLab: Ferramenta de Autoria para produção de OAs profissionais, não exigindo conhecimento em programação; Apresenta flexibilidade de uso; Permite resultado avançado para usuários familiarizados na edição de OAs; Permite alteração do template e eficiência para produção do OA.

2. eXe Learning: Ferramenta de Autoria com maior índice de flexibilidade para movimentação dos assets. Permite adaptação do conteúdo em diversos formatos; Permite edição HTML do conteúdo; Permite criar modalidades de recursos ou iDevices e apresenta semelhança em edição de conteúdo ao Moodle.

3. MyUdutu: Ferramenta de Autoria web que permite flexibilidade razoável na alteração do template, não exige conhecimento em programação ou familiaridade em edição de OA; Necessariamente o usuário produz o $\mathrm{OA}$ em diversas páginas, pois o tamanho da página é prefixado, não permitindo inserir deferentes recursos na mesma tela, por exemplo, o OA foi produzido com as três animações em flash em páginas distintas.

4. HotPotatoes: Ferramenta de Autoria para atividades e auto-avaliações; Permite razoável edição do formato do conteúdo, porém o OA não apresenta semelhança no layout do OA-R produzida em flash.

5. Xerte: Ferramenta de Autoria com template préfixado, apresentou distorções nas animações fla$s h$; Apresenta recursos não utilizados neste arti- 
go; Apresenta baixa flexibilidade em movimentação dos assets e permite baixa adaptação do conteúdo.

6. LCDS: Ferramenta de Autoria eficiente para produção dos OAs simples, porém com pouca adaptação do contudo e flexibilidade para movimentação dos assets na página do OA; Permite a gestão de erros de modo razoável, porém, para importação para Moodle a FA não obteve sucesso.

O presente artigo obteve como resultado dos três métodos avaliativos as seguintes médias: (a) eXeLearning com 93,54\%, (b) CourseLab com 90,42\%, (c) MyUdutu com 69,71\%, (d) HotPotatoes com 66,67\%, (e) Xerte com $64,79 \%$ e (e) $L C D S$ com $40,42 \%$.

A partir dos resultados concluímos que é possível a produção de OAs de qualidade, semelhante a um OA produzido pela equipe de DI\&DG, onde são utilizadas ferramentas profissionais de edição de conteúdos.

Futuros trabalhos visam a integração entre uma Ferramenta de Autoria avaliada e Ambiente Virtual de Ensino e Aprendizagem, e utilizar o método para avaliação heurística apresentado no presente artigo para realizar pesquisa com os usuários.

\section{Referências}

[1] Universidade Aberta do Brasil. Disp. em: <http://uab.capes.gov.br>. Acesso em: 20/04/ 2010 .

[2] Universidade Aberta do Sistema Único de Saúde (UnA-SUS). Disponível em:

<http://www.unasus.net>. Acesso em 20/04/2010.

[3] S. Ainshworth e P. Fleming. Evaluating Authoring Tools for Teachers as Instructional Designers. Comp in Human Behavior. 22: 131-148. Jan. 2006.

[4] P. E. Battistella et al. Classificação de Objetos de Aprendizagem e análise de Ferramentas de Autoria; XX Simpósio Brasileiro de Informática na Educação, 2009.

[5] J. Nielsen. Ten Usability Heuristics. Disponível em:

$<$ http://www.useit.com/papers/heuristic/heuristic_ list.html>. Acesso em 10/04/ 2010.

[6] J. M. C. Bastien, D. Scapin. Ergonomic Criteria for the Evaluation of Human-Computer Interfaces. Institute National de Recherche en Informatique et en Automatique, France, 1993.
[7] A. Zapata, V. H. Menendez e M. E. Prieto, Discovering Learning Objects Usability Characteristics. Intelligent Systems Design and Applications, International Conference on, 1126-1130, 2009 Ninth International Conference on Intelligent Systems Design and Applications, 2009.

[8] A. Segura, C. Vidal, V. Menedez, A. Zapata, M. Prieto. Exploring Characterizations of Learning Object Repositories Using Data Mining Techniques; Springer Berlin Heidelberg, 46: 215-225.

[9] Kaambal: Teleaprendizaje y Gestión del Conocimento. Disponível em:

$<\mathrm{http}$ ///sites.google.com/site/redkaambal/agora/re cursos-1>. Acesso em 15/03/2010.

[10] R. R. Relvão. Estudo Sobre a Utilização e Interoperabilidade entre Conteúdo de Aprendizagem com Diferentes Granularidades. Dissertação de Mestrado em Sistema de Informação. Universidade do Minho, Jun 2006.

[11] S. Downes. Learning Objects: Resourse for distance education worldwide. International Review of Research in Open and Distance Learning. 2, 1, 2001.

[12] T. Boyle. Design principles for authoring dynamic, reusable learning objects. Australian Journal of Educational Technology. 19: 46-58, 2003.

[13] J. H. Liu et al. The Design of Learning Objetc Authoring Tool Based on SCORM. Proceedings of the Fifth IEEE International Conference on Advanced Learning Technologies. 778-782, 2005.

[14] CourseLab. Disponível em: $<$ http://www.courselab.com>. Acesso em: 31/03/2010.

[15] eXe Learning. Disponível em: $<$ http://www.exelearning.org/wiki>. Acesso em $31 / 03 / 2010$.

[16] HotPotatoes. Disp.: <http://hotpot.uvic.ca/>. Acesso em: 31/03/2010.

[17] Microsoft LCDS. Disponível em: $<$ http://www.microsoft.com/learning/en/us/trainin g/lcds.aspx>. Acesso em: 31/03/2010.

[18] MyUdutu. Disponível em: <www.myudutu.com/>. Acesso em 31/03/2010.

[19] Xerte. Disponível em: $<$ http://www.nottingham.ac.uk/xerte/>. Acesso em: 31/03/2010.

[20] T. Murray, S. Ainsworth, e S. Blessing. Authoring Tools for Adv. Tech. Learning Env. Kluwer Academic Publishers. Printed in the Netherlands. 
493-546, 2003.

[21] I. Granchev, M. O’Droma e R. Andreev. Functionality and SCORM-compliancy Evaluation of eLearning Tools. Advanced Learning Technologies. Seventh IEEE Internacional Conference. 467-469, 2007.

[22] E. Kurilovas. Evaluation and Optimisation of eLearning Software Packages: Learning Object Repositories. Software Engineering Advances, International Conference on, 477-483, 2009 Fourth International Conference on Software Engineering Advances, 2009.

[23] J. Nielsen. Usability Engineering. Academic Press Limited. Boston, USA, 1993.

[24] M. J. M. da Ponte, A. M. da. A Methodology for Evaluation the Usability of Software for Industrial Automation Using Artificial Neural Networks: Case Study-Eletrobras. International Conference on Computational Intelligence for Modelling, Control and Automation. IEEE Computer Society. Los Angeles, CA, USA. 430-435, 2008.

[25] A. P. Gladcheff, A. M. Zuffi, D. M. da Silva. Um Instrumento para Avaliação da Qualidade de Softwares Educacionais de Matemática para o Ensino Fundamental. Congresso da Sociedade Brasileira de Computação. VII Workshop de Informática na Escola, Fortaleza, CE, Brasil, 2001.

[26] E. García-Barriocanal, M. A. Sicilia, M. Lytras. Evaluating pedagogical classification frameworks for learning objects: A case study. Computers in Human Behavior, Including the Special Issue: Education and Pedagogy with Learning Objects and Learning Designs. 23, 6, 2641-2655, 2007. 